International Journal of Pure and Applied Mathematics

Volume 96 No. 4 2014, 445-456

ISSN: 1311-8080 (printed version); ISSN: 1314-3395 (on-line version)

url: http://www.ijpam.eu

doi: http://dx.doi.org/10.12732/ijpam.v96i4.3

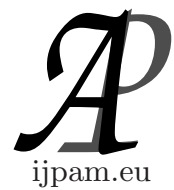

\title{
THE MODIFIED VARIATIONAL ITERATION METHOD FOR SOLVING THE IMPENETRABLE AGAR MODEL PROBLEM
}

\author{
Ameina S. Nuseir ${ }^{2} \S$, Mohammad Al-Towaiq ${ }^{2}$ \\ ${ }^{1,2}$ Department of Mathematics and Statistics \\ Jordan University of Science and Technology \\ P.O. Box 3030, Irbid 22110, JORDAN
}

\begin{abstract}
In this paper, we expand the scope of application of the modified variational iteration method (MVIM) to solve the Impenetrable Agar Model Problem. This model represents the propagation of a bacterial exo-enzyme within an impenetrable host material which simulates the manifestations bacteria do on tissue wounds in an infection. The numerical results show that the MVIM is convenient, efficient, and easy to use for the model problem.
\end{abstract}

AMS Subject Classification: $35 \mathrm{~A} 15,74 \mathrm{G} 15,74 \mathrm{H} 15$

Key Words: variational iteration method, modified variational iteration method, impenetrable agar model problem, numerical solutions

\section{Introduction}

In 1978 Inokuti et al. [1] proposed a general Lagrange multiplier method to solve nonlinear problems. Ji-huan He modified the method of Inokuti and proposed the VIM [2- 6]. This method has been applied to solve a large class of linear and nonlinear problems, and proved by many researchers to be reliable, efficient, and convenient for a variety of scientific applications [7-19].

On the other hand, the VIM has also been applied to solve fractional dif-

Received: December 14, 2013

(C) 2014 Academic Publications, Ltd.

$\S$ Correspondence author url: www.acadpubl.eu 
ferential equations. Soltani and Shirzadi [20] proposed a new modification of the VIM; the given examples show that the method provides great freedom in choosing linear operators for various nonlinear equations which leads to identify the Lagrange multipliers effectively. Geng [21], proposed a modification

of the VIM for solving Riccati differential equations, where he compared his modification with the standard VIM.

This work uses the modified method (MVIM) to solve the propagation of the bacteria through wounds and the manipulations it performs to degrade wound tissue. The given example shows that the modified method gives a more accurate approximation in a large region and overcome the restriction of the application area of the VIM. Complete review of He's VIM can be found in references 5 and $22[5,22]$.

This paper is organized as follows: In Section 2 we introduce the VIM and its modification, in Section 3 we discuss the Impenetrable Agar Model Problem, where in Section 4 we implement and analyze the method for the solution of the model problem using the MVIM, and finally we conclude the paper in Section 5 .

\section{The Variational Iteration Method and the Modified Variational Iteration Method}

In this section we introduce the He's VIM [2-5].

Consider the differential equation

$$
L u+N u=g(x),
$$

where $L$ is a linear operator, $N$ a nonlinear operator and $g(x)$ is the inhomogeneous term. J. He proposed the VIM by constructing a correction functional for equation 1 as follows:

$$
u_{n+1}(x)=u_{n}(x)+\int_{0}^{x} \lambda(t)\left(L u_{n}(t)+N \breve{u}_{n}(t)-g(t)\right) d t,
$$

where $\lambda$ is a Lagrange's multiplier and it can be constant or a function, and $\breve{u}_{n}$ as a restricted variation, we can assume that the correction functional is stationary, that is $\partial \breve{u}_{n}=0$. In this method we can construct a correction functional form using the general Lagrange multiplier which can be identified by the variational theory [4]. Then the iterative approximation can start with initial approximation $u_{0}$ which can be obtained from the initial conditions $\mathrm{u}(0)$, 
$u^{\prime}(0)$, and $u^{\prime \prime}(0)$. The exact solution may be obtained by taking the limit as $n \rightarrow \infty$, that is $u(x)=\lim _{n \rightarrow \infty} u_{n}(x)$.

In 2010 Soltani and Shirzadi [20], presented a very simple and effective modification of the VIM which is based on introducing an arbitrary linear operator $L 1[u(x)]$ as follows:

1. Rewrite equation 1 in the form:

$$
L[u(x)]+L_{1}[u(x)]-L_{1}[u(x)]+N[u(x)]=g(x),
$$

where $L_{1}[u(x)]$ is an arbitrary linear operator of $u(x)$.

2. Construct the correction functional

$$
u_{n+1}(x)=u_{n}(x)+\int_{0}^{x} \lambda(t)\left(L u_{n}(t)+L_{1}\left[u_{n}(x)\right]-L_{1}\left[\breve{u}_{n}(x)\right]+N \breve{u}_{n}(t)-g(t)\right) d t,
$$

where $\breve{u}_{n}$ is considered as a restriction variation.

According to Theorem 1 of Section 2 in [20], a sufficient condition for convergence of the MVIM is strictly contraction of $A$, where $u_{n+1}(x)=A\left[u_{n}\right]$, which follows that the sequence $u_{n+1}(x)=A\left[u_{n}\right]$,converges to the solution of equation 1.

\section{The Impenetrable Agar Model Problem}

In 2003 King et al [23], studied a model in which a bacterial colony is unable to penetrate the 'tissue' (agar substrate), and must obtain nutrients by producing exo-proteases to break down the milk protein contained in an agar matrix. They were trying to simulate the propagation of the bacteria ( $P$. aeruginosa) through wounds and the manipulations it performs to degrade wound tissue. This model is a simplified model for the propagation of the bacteria due to the fact that it does not involve the complications occurs within the tissue. Hence, their case provides useful insights and validation of the introduced modeling approach they presented, and the simplified model lends itself to a detailed mathematical analysis. In their experiment, an impenetrable matrix of agar infused with dissolved milk powder was constructed to provide a simplified system in which $P$. aeruginosa was unable to move into the substrate but produces exo-proteases in order to degrade milk proteins into utilizable peptides for its growth. 
The simplest model they used is

$$
\begin{gathered}
\frac{\partial c}{\partial t}=D \nabla^{2} c-\lambda c-\alpha c m \\
\frac{\partial m}{\partial t}=-\beta c m
\end{gathered}
$$

where the concentration of the most active bacterial enzyme is denoted by $c$, and that of milk by $m$. While the constants $\alpha, \beta, \lambda$ and $D$ respectively denote the rates of bacterial enzyme,

Milk used up in the reaction, the natural inactivation rate of the exoenzyme, and the rate of diffusion of the exo-enzyme within the agar. Hence, the milk is thus assumed to be immobile until it is degraded by the enzyme to produce diffusible peptides.

They considered a semi-infinite region of substrate, with $z$ defined as positive in the downward direction (into the agar) with a disc-shaped colony of bacteria deposited on the surface. Hence the initial data becomes

$c=0, m=m_{i}$ at $t=0, z>0$,

where $m_{i}$ is the initial concentration of milk protein, and boundary conditions

$$
c=c_{b} \text { on } z=0, x^{2}+y^{2}<l^{2} \text { and } \frac{\partial c}{\partial z}=0 \text { on } z=0, x^{2}+y^{2}>l^{2},
$$

which correspond to the surface bacterial layer suddenly producing enzymes at a constant level $c b$ on the boundary and then continuing to hold that level. This sudden switch is an exaggerated version of the quorum sensing-induced jump in virulence determinant production that does occur in P.aeruginosa.

Therefore, the dimensionless model will be

$$
\begin{gathered}
\frac{\partial \hat{c}}{\partial \hat{t}}=\nabla^{2} \hat{c}-k \hat{c}-\frac{\gamma}{\varepsilon^{2}} \hat{c} \hat{m} \\
\frac{\partial \hat{m}}{\partial \hat{t}}=-\frac{1}{\varepsilon^{2}} \hat{c} \hat{m}
\end{gathered}
$$

where,

$$
\hat{m}=\frac{m}{m_{i}}, \hat{c}=\frac{c}{c_{b}}, \hat{t}=\frac{D}{l^{2}} t, \hat{\mathrm{x}}=\frac{1}{l} \mathrm{x}
$$

immediately dropping the hats will give

$$
\frac{\partial c}{\partial t}=\nabla^{2} c-k c-\frac{\gamma}{\varepsilon^{2}} c m
$$




$$
\frac{\partial m}{\partial t}=-\frac{1}{\varepsilon^{2}} c m
$$

with initial condition

$c=0, m=1$ at $t=0, z>0$

and boundary conditions

$c=1$ on $z=0, x^{2}+y^{2}<1$ and $\frac{\partial c}{\partial z}=0$ on $z=0, x^{2}+y^{2}>1$.

The dimensionless parameters are

$\varepsilon=\sqrt{\frac{D}{\beta c_{b} l^{2}}}, \gamma=\frac{\alpha m_{i}}{\beta c_{b}}$, and $k=\frac{\lambda l^{2}}{D}$.

\section{The Solution Method and Analysis}

In this section, we implement and analyze the MVIM for solving the model problem in equations 3 and 4 when $x^{2}+y^{2}>1$.

Consider the following correction functional for $c$ and $m$ respectively:

$$
\begin{gathered}
\delta c_{n+1}(t)=\delta c_{n}(t)+\delta \int_{0}^{t} \lambda_{1}(\tau)\left(-\frac{\partial^{2} c_{n}}{\partial \tau^{2}}+\frac{\partial \tilde{c}_{n}}{\partial \tau}-\frac{\partial^{2} \tilde{c}_{n}}{\partial z^{2}}+\kappa \tilde{c}_{n}+\frac{\gamma}{\varepsilon^{2}} \tilde{c}_{n} \tilde{m}_{n}\right) d \tau \\
\delta m_{n+1}(t)=\delta m_{n}(t)+\delta \int_{0}^{t} \lambda_{2}(\tau)\left(\frac{\partial m_{n}}{\partial \tau}+\frac{1}{\varepsilon^{2}} \tilde{c}_{n} \tilde{m}_{n}\right) d \tau
\end{gathered}
$$

which leads to the following

$$
\begin{aligned}
& \delta c_{n+1}(t)=\delta c_{n}(t)+\delta \int_{0}^{t} \lambda_{1}(\tau)\left(-\frac{\partial^{2} c_{n}}{\partial \tau^{2}}\right) d \tau, \text { and } \\
& \delta m_{n+1}(t)=\delta m_{n}(t)+\delta \int_{0}^{t} \lambda_{2}(\tau) \frac{\partial m_{n}}{\partial \tau} d \tau . \text { Since } \delta \tilde{c}_{n}=0, \text { and } \delta \tilde{m}_{n}=0 .
\end{aligned}
$$

The extremum conditions of $c_{n+1}$ and $m_{n+1}$ requires that $\delta c_{n+1}(t)=0$, and $\delta m_{n+1}(t)=0$. Doing this will lead to the stationary conditions for $\lambda_{1}$ and $\lambda_{2}$

$$
\begin{aligned}
& 1+\left.\lambda_{1}^{\prime}\right|_{\tau=t}=0, \\
& \left.\lambda_{1}\right|_{\tau=t}=0, \\
& \lambda_{1}^{\prime \prime}=0 . \\
& 1+\left.\lambda_{2}\right|_{\tau=t}=0, \\
& \lambda_{2}^{\prime}=0 .
\end{aligned}
$$

The solutions of 7 and 8 implies that $\lambda_{1}=t-\tau$, and $\lambda_{2}=-1$.

Substituting this value of the Lagrange multiplier into the functional 5 and 6 gives the iteration formulae: 


$$
\begin{gathered}
c_{n+1}(t)=c_{n}(t)+\int_{0}^{t}(t-\tau)\left(\frac{\partial c_{n}}{\partial \tau}-\frac{\partial^{2} c_{n}}{\partial \tau^{2}}-\frac{\partial^{2} c_{n}}{\partial z^{2}}+\kappa c_{n}+\frac{\gamma}{\varepsilon^{2}} c_{n} m_{n}\right) d \tau \\
m_{n+1}(t)=m_{n}(t)-\int_{0}^{t}\left(\frac{\partial m_{n}}{\partial \tau}+\frac{1}{\varepsilon^{2}} c_{n} m_{n}\right) d \tau .
\end{gathered}
$$

By choosing the initial approximation $c_{0}(t, z)=t \operatorname{Cosh}[z]$, and $m_{0}(t, z)=1$, which satisfies the initial condition of the original problem $c=0, m=1$ at $t$ $=0, z>0$, and the boundary condition $\frac{\partial c}{\partial z}=0$ at $z=0$.

Applying the iteration formula, will give the following first approximation

$$
c_{1}(t, z)=\frac{1}{6} t\left(6+3 t+\left(-1+\frac{b}{c^{2}}+k\right) t^{2}\right) \operatorname{Cosh}[z]
$$

and

$$
\mathrm{m}_{1}(\mathrm{t}, \mathrm{z})=1-\frac{\mathrm{t}^{2} \operatorname{Cosh}[\mathrm{z}]}{2 \mathrm{c}^{2}} .
$$

And the second iteration of " $c_{2}$ " and " $m_{2}$ " can be obtained from 9 and 10;

$$
\begin{aligned}
c_{2}=\frac{1}{2520 c^{6}} t \operatorname{Cosh}(z) \\
\left(\begin{array}{l}
21\left(b^{2} c^{2} t^{4}+2 b c^{4} t^{2}\left(10+5 t+(-1+k) t^{2}\right)+\right. \\
\left.c^{6}\left(120+60 t+20 k t^{2}+10(-1+k) t^{3}+(-1+k)^{2} t^{4}\right)\right)- \\
b t^{4}\left(5 b t^{2}+c^{2}\left(63+21 t+5(-1+k) t^{2}\right)\right) \operatorname{Cosh}(z)
\end{array}\right)
\end{aligned}
$$

and

$$
\begin{gathered}
m_{2}=1-\frac{t^{2}\left(b t^{2}+c^{2}\left(12+4 t+(-1+k) t^{2}\right)\right) \operatorname{Cosh}(z)}{24 c^{4}} \\
+\frac{t^{4}\left(5 b t^{2}+c^{2}\left(45+18 t+5(-1+k) t^{2}\right)\right) \operatorname{Cosh}^{2}(z)}{360 c^{6}}
\end{gathered}
$$

The following two Figures represent the third iteration of the approximate solutions of $m_{3}$ and $c_{3}$ respectively:

From the above we notice that all iterations satisfy the initial and the boundary conditions and we conclude that the third iteration of MVIM is a reasonable approximation for the solution of the impenetrable agar model problem. These figures show that the concentration of milk " $m$ " decreases as time increases. 


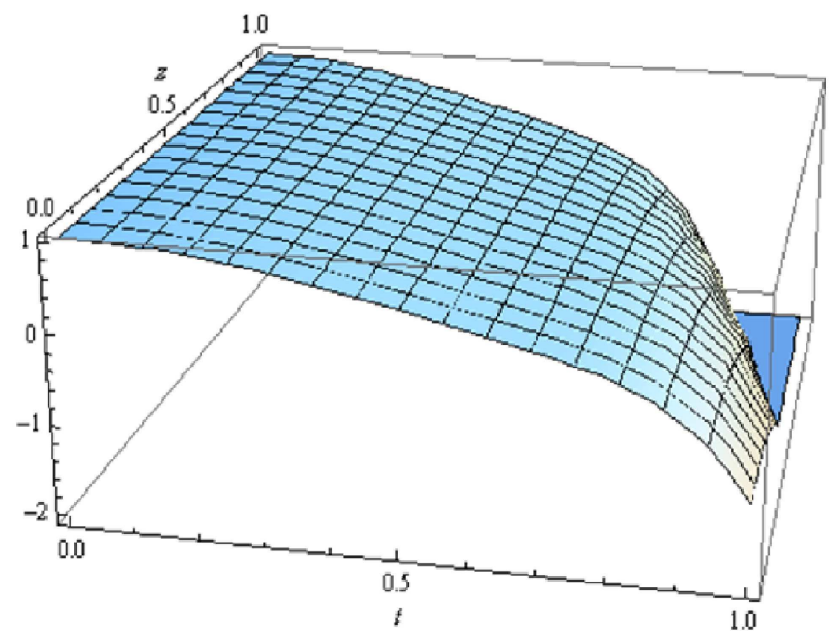

Figure 1: Depicts the approximate solution of $m_{3}$ when $c=0.5, k=1$ and $b=1$

While the concentration of the enzyme increases with time. This demonstrates a reasonable explanation of the degradation of milk in the presence of bacteria as time increases.

\section{Solving the Impenetrable Agar Model Problem Using Variational Iteration Algorithm II}

In this section a comparison between the results obtained for the Impenetrable Agar Model Problem using algorithm II and that obtained in the previous section. He [11] summarized three variational iteration algorithms. The variational iteration method mentioned in section two is called variational iteration 


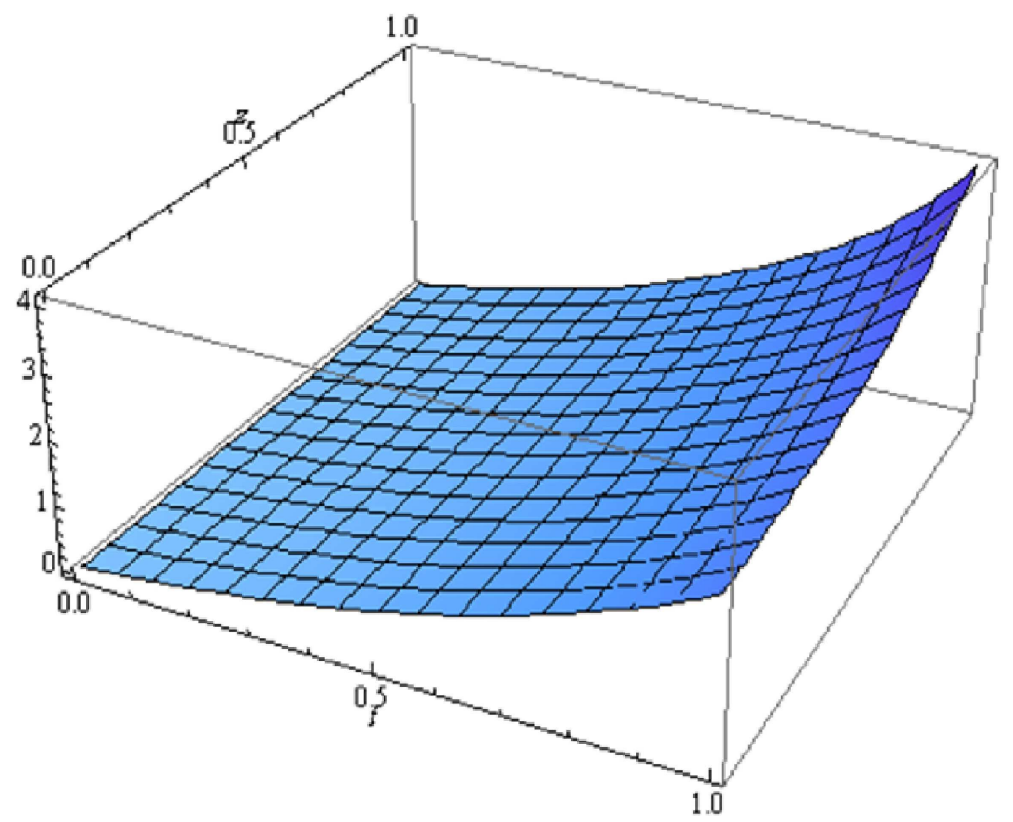

Figure 2: Depicts the approximate solution of $c_{3}$ when $c=0.5, k=1$ and $b=1$

algorithm I. The variational iteration algorithm II

$$
u_{n+1}(x)=u_{0}(x)+\int_{0}^{x} \lambda(t)\left(N \breve{u}_{n}(t)-g(t)\right) d t,
$$

was used to construct a solution for the problem of concern. The algorithm becomes

$$
\begin{gathered}
c_{n+1}(t)=c_{n}(t)+\int_{0}^{t}(t-\tau)\left(-\frac{\partial^{2} c_{n}}{\partial \tau^{2}}-\frac{\partial^{2} c_{n}}{\partial z^{2}}+\kappa c_{n}+\frac{\gamma}{\varepsilon^{2}} c_{n} m_{n}\right) d \tau \\
m_{n+1}(t)=m_{n}(t)-\int_{0}^{t}\left(\frac{1}{\varepsilon^{2}} c_{n} m_{n}\right) d \tau .
\end{gathered}
$$

The following figures show the comparison between the solutions obtained using algorithm II and that obtained by the modified method. Figure 3 display 
the third iteration $c_{3}$ when $\gamma=\kappa=1$ with different values for $\varepsilon$. Figure 3(A) represent the solution when $\varepsilon=0.5$, where $\mathrm{z}=0.6$. In (B) $\varepsilon=0.5$, and $z=0.3$. In $(\mathrm{C})$ the value for $\varepsilon=0.8=z$. While in $(\mathrm{D}) \varepsilon=0.8$, and $z=0.7$.
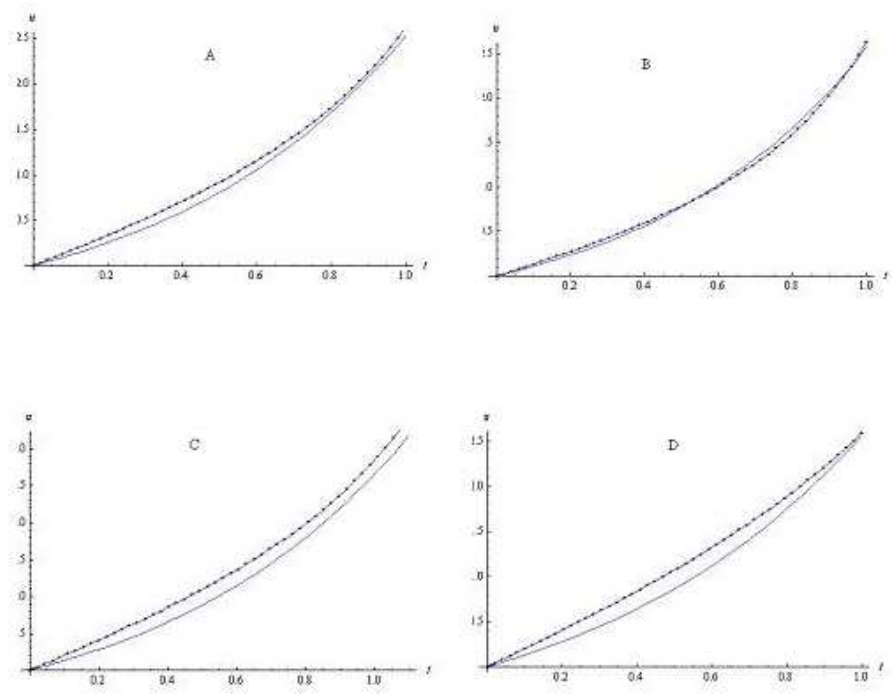

Figure 3: The mesh curve represents the approximate solution of $c_{3}$ using algorithm II, while the second curve represent the approximate solution of $c_{3}$ using the modified method when $\kappa=1$ and $\gamma=1$.

Figure 4 shows the approximate solutions of $m_{3}$ for $\gamma=\kappa=1$ with different values for $\varepsilon$. Figure 4 (A)represent the solution when $\varepsilon=0.8$, where $z=1.0$. In $(\mathrm{B}) \varepsilon=0.7$, and $z=0.9$. In $(\mathrm{C})$ the value for $\varepsilon=1.0$, and $z=1.4$. While in (D) $\varepsilon=0.8$, and $z=0.7$.

\section{Conclusion}

In this paper, we have applied the MVIM for solving the Impenetrable Agar Model Problem. The numerical results show that the method converges and provides powerful tools for the solution of the model problem and gives a reasonable explanation of the degradation of milk in the presence of bacteria as time increases. 

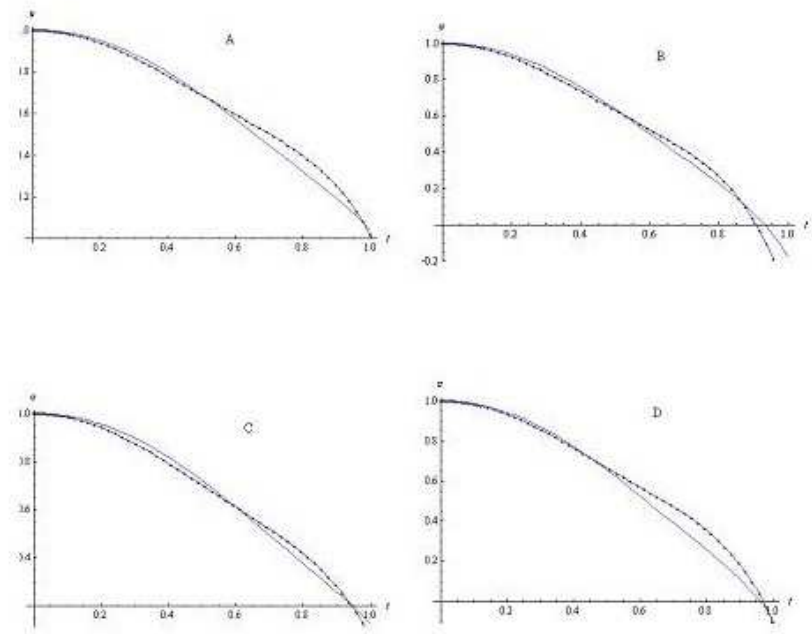

Figure 4: The mesh curve represents the approximate solution of $m_{3}$ using algorithm II, while the second curve represent the approximate solution of $m_{3}$ using the modified method when $\kappa=1$ and $\gamma=1$

\section{References}

[1] M. Inokuti, H. Sekine and T. Mura, General use of the Lagrange multiplier in nonlinear mathematics, in Variational Method in Mechanics of Solids, Nemat-Nassers (Ed.), Pergamon Press, Oxford, 156-162 (1978).

[2] Ji-huan He, Varitional iteration method- a kind of non-linear analytical technique: some examples, International Journal of Non-Linear Mechanics, 34,(1990), 699-708.

[3] Ji-huan He, A New Approch to Nonlinear Partial Differential Equations, Communication in Nonlinear Science 83 Numerical Simulation, 2, No.4 (1997), 230-235.

[4] Ji-huan He, Variational iteration method some recent results and new interpretations, J. Comput. Appl. Math., 207, (2007), 3-17.

[5] Ji-huan He, Some asymptotic methods for strongly nonlinear equations, Int. J. Modern Phys., 20, (2006), 1141-1199. 
[6] B. Batiha, M. S. M. Noorani, I. Hashim, Numerical Solutions of the Nonlinear Integro-Differential Equations, Int. J. Open Problems Compt. Math. , 1, (2008), 34-42.

[7] A. Elsaid, The variational iteration method for solving Riesz fractional partial differential equation, Computers and Mathematics with Applications, 60, (2010), 1940-1947.

[8] A. Ghorbani and A. Alavi, Application of He's Variational Iteration Method to Solve Semidifferential Equations of nth Order, Mathematical Problems in Engineering, 2008, (2008), Page Article ID 627983.

[9] H. Jafari, H. Hosseinzadeh, and E. Salehpoor, A New Approach to the Gas Dynamics Equation: An Application of Varitional Iteration Method, Applied Mathematical Sciences, 2, No. 48, (2008), 2397-2400.

[10] R. Saadati, B. Raftari, H. Abibi, S.M. Vaezpour, and S. Shakeri, A Comparison Between the Varitional Iteration Method and Trapezoidal Rule for Solving Linear Integro-Differential Equations, World Applied Sciences Journal , 4, No. 3, (2008), 321-325.

[11] Ji-huan He and Xu-Hong Wu, Variational iteration method: new development and applications, Computers and Mathematics with Applications, 54, (2007), 881-894.

[12] Ji-huan He, Approximate analytical solution for seepage with fractional derivitaves in porous media, Compt. Methods Appl. Mech. Engrg., 167, (1998), 57-68.

[13] S. Das, Analytical Solution of Fractional Diffusion Equation by Variational Iteration Method, Comput. Math. Appl., 57, (2009), 483-487.

[14] A. Wazwaz, The variational iteration method for rational solutions for $\mathrm{KdV}, K(2,2)$, Burgers, and cubic Boussinesq equations, Journal of Computational and Applied Mathematics, 207, (2007), 18-23.

[15] A. Wazwaz, A comparison between the variational iteration method and Adomian decomposition method, Journal of Computational and Applied Mathematics, 207, (2007), 129-136.

[16] A. Wazwaz, The variational iteration method for solving two forms of Blasius equation on a half-infinite domain, Applied Mathematics and Computation, 188, No. 1, (2007), 485-491. 
[17] A. Wazwaz, The variational iteration method for exact solutions of Laplace equation, Physics Letters A, 363, (2007), 260-262.

[18] A. Wazwaz, The variational iteration method; a reliable tool for solving linear and nonlinear wave equations, Computers and Mathematics with Applications, 54, (2007), 926-932.

[19] A. Wazwaz, The variational iteration method for solving systems of equations of Emden-Fowler type, International Journal of Computer Mathematics, 82, No. 9, (2011), 1107-1115.

[20] L. A. Soltani and A. Shirzadi, A new modification of the variational iteration method, Computers and Mathematics with Applications, 59, (2010), 2528-2535.

[21] F. Geng, A modified variational iteration method for solving Riccati differential equations, Computers and Mathematics with Applications, 60, (2010), 1868-1872.

[22] S. T. Mohyud-Din, M. A. Noor and K. I. Noor, Some Relatively New Techniques for Nonlinear Problems, Mathematical Problems in Engineering, 2009, (2009), Article ID 234849.

[23] J. R. King, A. J. Koerber, J. M. Croft, J. P. Ward, P. Williams and R. E. Sockett, Modelling host tissue degradation by extracellular bacterial pathogens, Mathematical Medicine and Biology, 20, (2003), 227-260. 